\title{
Study of an Ultrasonic Probe Installed into a Small Diameter-Pipe Using an Electromagnetic Acoustic Transducer
}

\author{
Riichi Murayama, Benjie Wang, Koutarou Shindou, Koudai Katsunaga \\ Fukuoka Institute of Technology, Fukuoka, Japan \\ Email:murayama@fit.ac.jp
}

How to cite this paper: Murayama, R., Wang, B.J., Shindou, K. and Katsunaga, K. (2020) Study of an Ultrasonic Probe Installed into a Small Diameter-Pipe Using an Electromagnetic Acoustic Transducer. Engineering, 12, 549-562.

https://doi.org/10.4236/eng.2020.128038

Received: July 14, 2020

Accepted: August 11, 2020

Published: August 14, 2020

Copyright $\odot 2020$ by author(s) and Scientific Research Publishing Inc. This work is licensed under the Creative Commons Attribution International License (CC BY 4.0).

http://creativecommons.org/licenses/by/4.0/

\section{(c) (i) Open Access}

\begin{abstract}
Metal pipes having an inner diameter of about $25 \mathrm{~mm}$ or less are frequently used as heat exchangers for power plants, gas pipes, and water pipes. However, erosion and corrosion due to long-term use may cause serious accidents, such as steam leaks, resulting in economic loss and environmental pollution. Therefore, inspection of the entire length and thickness before shipping or monitoring during operation are important technologies. However, no inspection technology including the inside of the wall thickness has been developed. The purpose was to develop an ultrasonic probe that can inspect the inner and outer surfaces from the inside of the pipe at the same time. The developed ultrasonic probe is based on an electromagnetic ultrasonic transducer (EMAT) that does not require a couplant and is then easy to install in a pipe. The EMAT for the longitudinal and for the transverse vibration mode guided wave are connected in series in order to take into account the variety of defects. First, the EMAT was successfully developed for each mode. That is, it was conducted by using the magnetostrictive effect for the longitudinal mode type and by using the Lorentz force for the transverse mode type, and evaluated to improve the performance. The reflected signal from a notch defect was then evaluated in the state that each EMAT was connected in series using any artificial defects and found to be able to detect any notches with about $10 \%$ depth or about $15 \%$ circumferential length.
\end{abstract}

\section{Keywords}

EMAT, Small Diameter Pipe, Nondestructive Inspection, Guide Wave

\section{Introduction}

Pipes are one of the main transport tools and are the main means of transporting liquids, gases and slurries. For example, the main trunk of gas pipes around the 
world has reached 2,300,000 km. However, these pipes are used for a long time, and the wall of the pipe becomes thin due to corrosion, possibly causing a leak and subsequent accident [1] [2] [3]. Not only does it cause significant economic losses, but it also affects the balance of the environment and ecology.

Non-destructive inspection is a method that uses variables, such as ultrasonics, light, eddy current, and X-rays to determine whether a defect or non-uniformity exists in a test object, assuming that the test object is not destroyed. Non-destructive inspection is an effective means to guarantee the quality of materials and structures, then realize quality control in society. With the increasing emphasis on environmental protection and safety, non-destructive inspection is widely used in industries, such as railways, ships, metallurgy, and machinery manufacturing, has significant economic effects, and has attracted the attention of many researchers [4] [5] [6].

The inspection of pipes using conventional non-destructive inspection methods has many advantages. For example, as non-destructive inspection techniques mature, workers can do inspections using existing specialized equipment with little training. However, inspection is basically a method of inspecting each point one by one, so it is not effective for the inspection of the entire length of a pipe. While long pipes are becoming more and more widely applied in industry, they need to be quickly analysed using efficient and convenient non-destructive inspection methods. In a pipe inspection by ultrasonic waves, the guide wave propagating in the axial direction has been rapidly studied for application in recent years. The reason is that the guided wave can be inspected in the entire circumferential direction at one time, and the distance in which it can propagate in the axial direction is relatively long, so that the inspection efficiency is high. Another advantage is that the guided wave has various modes. That is, the vibration distribution in the circumferential direction and the thickness direction is different for each mode, so by using multiple modes, it is possible to detect any nochies of various shapes at various positions. As a study of guided waves propagating in pipes, a pipe inspection method by installing an ultrasonic sensor on the pipe surface of a pipe with a diameter of $60 \mathrm{~mm}$ or more has been studied by many research institutions and partially put into practical use. On the other hand, the pipes used in heat exchangers, such as power plants, are about $20 \mathrm{~mm}$ in diameter, and pipes are often installed adjacent to each other. It makes difficult to transmit and receive guided waves using the outer surface. In the past, the inspection from the other end of a pipe with an insertion probe based on the eddy current method was mainly used. However, the eddy current method had a weak point in its ability to detect internal defects. A probe inserted into a pipe using an EMAT for a guided wave has already been studied, but it has not been applied to pipes with a diameter of $20 \mathrm{~mm}$ or less, and simultaneous evaluation of flaw detection capability using multiple modes guide wave has not been performed. Therefore, in this study, a probe inserted into a pipe that applied to a small diameter pipe with a diameter of about $20 \mathrm{~mm}$, that uses both longitudinal 
and transverse mode guided waves at the same time, has been researched [7] [8] [9].

\section{Outline of Prototype Ultrasonic Sensor Installed in a Pipe}

Since an ultrasonic sensor using a piezoelectric vibrator requires a couplant, there are problems from the viewpoints of simplicity and reliability of the inspection results. Therefore, an electromagnetic ultrasonic sensor (EMAT) that does not require a couplant was used as the basic structure. An EMAT is a sensor that can directly generate ultrasonic waves on the surface of a metal specimen using an electromagnetic force. In ferromagnetic materials, the magnetostriction effect as well as the Lorentz force is the main cause of the generation and reception of ultrasonic waves. In many ferromagnetic materials, the magnetostriction effect always plays a leading role [10] [11] [12].

The prototype ultrasonic probe installed in a pipe consisting of two EMATs is shown in Figure 1. One can transmit and receive a longitudinal vibration mode guided wave mainly having a vibration component parallel to the tube axis direction. The other can transmit and receive a transverse vibration mode guided wave mainly having a vibration component vertical to the tube axis direction. The prototype ultrasonic probe could detect both circumferentially and axially extending flaws. Therefore, in the case of the longitudinal vibration mode guided wave, the EMAT generates a force that vibrates in the same axis direction of the pipe as the traveling direction, and in the case of the transverse vibration mode guided wave, the EMAT has a structure that generates a force that vibrates in the circumferential direction of the pipe. Figure 2 shows the relationship between the frequency and the group velocity in the case of the steel pipe with an outer diameter of $21.8 \mathrm{~mm}$ and a thickness of $2.8 \mathrm{~mm}$ used for the experiment.

Figure 2 shows the basic configuration of a prototype ultrasonic probe, in which a trans mission/reception EMAT for a longitudinal mode guided wave and a transmission/reception EMAT for a transverse mode guided wave are connected from one end of a pipe. Initially, the evaluation of EMAT for each mode guided wave was independently performed, then a simple flaw signal evaluation test was performed using an artificial flaw machined in a pipe after connection.

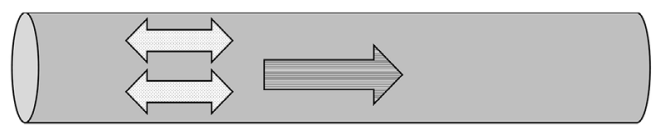

Longitudinal vibration mode guide wave

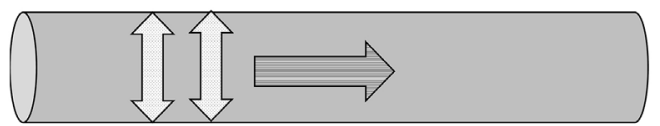

Transverse vibration mode guide wave
Oscillation direction

Traveling direction

Figure 1. Vibration pattern of a longitudinal vibration mode guided wave and transverse vibration mode guided wave. 
Longitudinal vibration guide wave

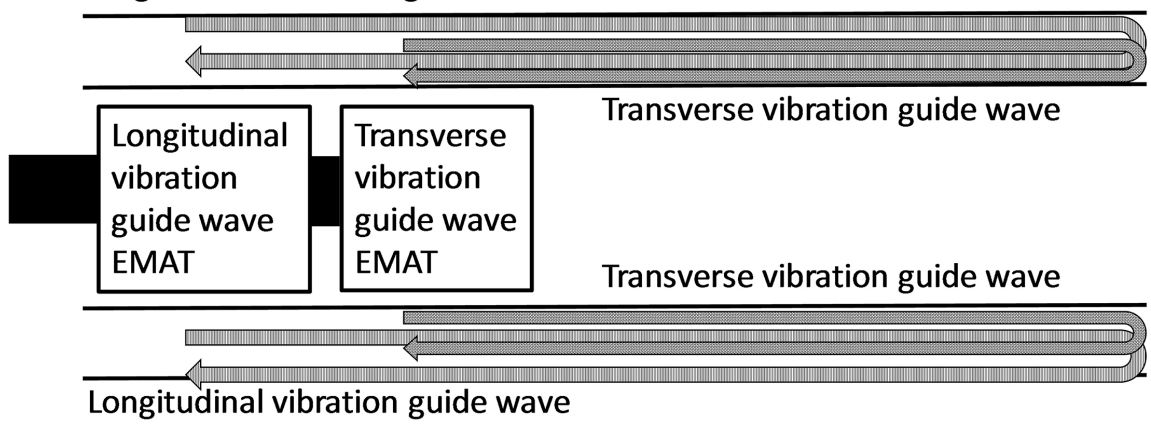

Figure 2. Outline of prototype ultrasonic interior probe.

\section{Decision of the Guide Wave Mode Considering Its Detectability Depends on the Oscillation Pattern to the Thick Direction}

A prototype of an ultrasonic probe was designed that transmits and receives a guided wave at a frequency of $250 \mathrm{kHz}$, where the group velocity of the $\mathrm{L}(0,2)$ mode corresponding to the longitudinal vibration mode guided wave is relatively stable. The group velocity in the $\mathrm{T}(0,1)$ mode corresponding to the transverse vibration mode guided wave is constant regardless of the driving frequency. To be sure, it is confirmed the vibration components of each guided wave mode at different drive frequencies were confirmed. The relationship between the distance from the surface of a pipe and the displacement amplitude for each vibration direction, in the case of the $\mathrm{L}(0,2)$ mode at a driving frequency of $250 \mathrm{kHz}$ (A), the $\mathrm{T}(0,1)$ mode at a driving frequency $250 \mathrm{kHz}(\mathrm{B})$, the $\mathrm{L}(0,2)$ mode at a driving frequency $(C)$, and $T(0,1)$ mode at a driving frequency $(D)$, are shown in Figure 3. Figure 4 shows the analysis results of the vibration pattern to the thickness direction. The $\mathrm{T}(0,1)$ mode has a vibration component only in the circumferential direction at the driving frequencies of $250 \mathrm{kHz}(\mathrm{B})$ and $1000 \mathrm{kHz}$ (D), and the distribution of the vibration component in the thickness direction is relatively uniform. In the $\mathrm{L}(0,2)$ mode at $250 \mathrm{kHz}(\mathrm{A})$, there is no circumferential component and the axial direction ( $\mathrm{Z}$ direction) is relatively uniformly distributed, and the radial component of the bottom surface is about $25 \%$ of the axial component on the pipe surface and converges to $0 \%$ toward the inside surface. Therefore, there is the possibility that information in the thickness direction cannot be more accurately evaluated than in the $\mathrm{T}(0,1)$ mode. In the case of the $\mathrm{L}(0,2)$ mode at the driving frequency of $1000 \mathrm{kHz}$, the circumferential vibration component does not exist, but the axial vibration component and the radial vibration component equally exist, and the distribution in the thickness direction is reversed. Since the drive frequency of $250 \mathrm{kHz}$ was selected for the experiment, it seems that the $\mathrm{L}(0,2)$ mode can be considered as a longitudinal vibration guided wave and the $\mathrm{T}(0,1)$ mode is a transverse vibration guided wave. The analysis results in Figure 3 and Figure 4 used the software (Pipe Dispersion Ver. 2.02) developed by Hayashi [13]. 


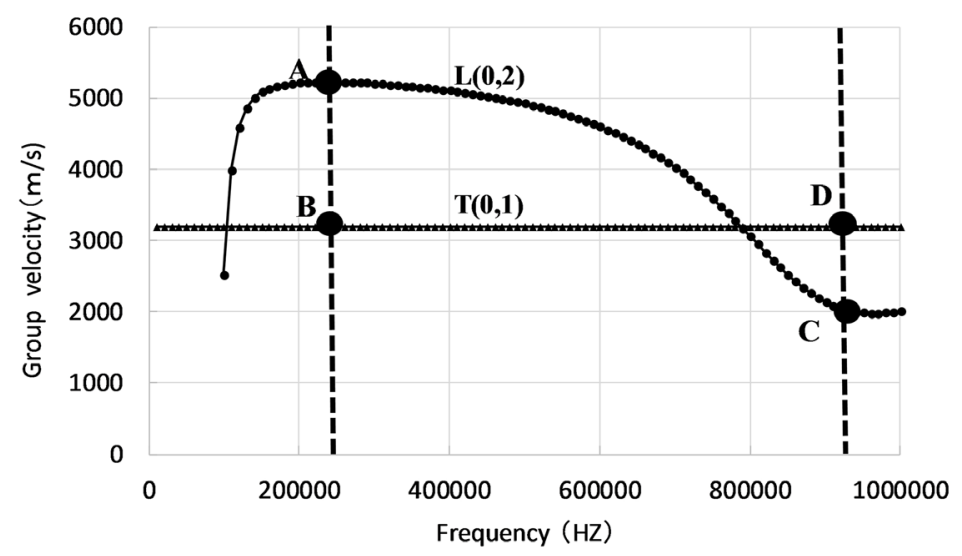

Figure 3. Relationship between group velocity and drive frequency in the case of a pipe of 21.8-mm diameter, and 2.8-mm thickness.
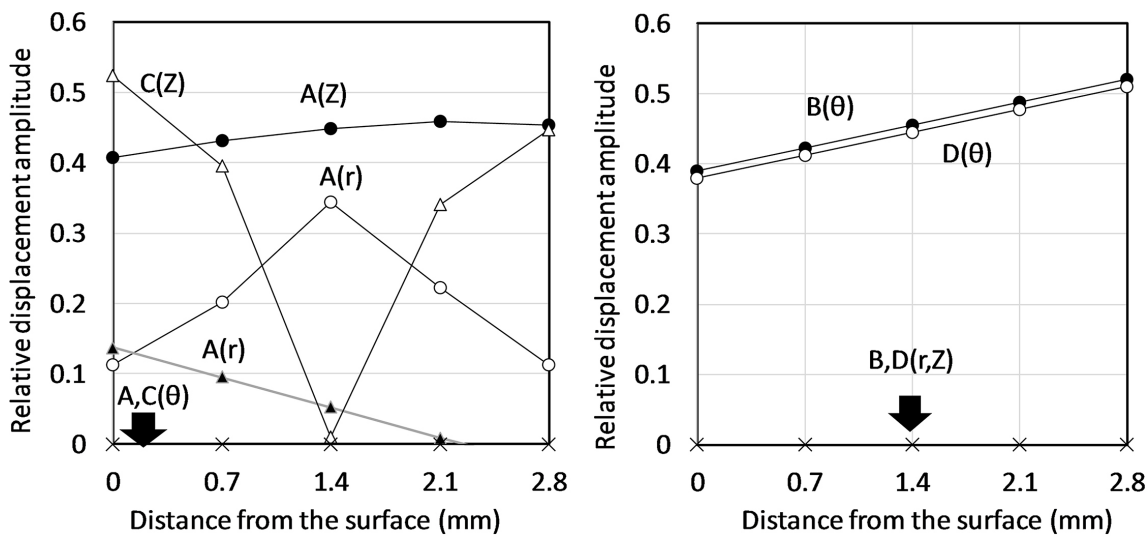

Figure 4. Relationship between vibration amplitude and position from pipe surface for each vibration direction.

\section{Experimental Condition}

First, the EMAT for the longitudinal vibration mode guided wave and the EMAT for the transverse vibration guided wave were individually optimized, and finally the combination experiment to evaluate the flaw detection ability was performed.

\section{Experimental System}

Figure 5 shows the experimental system in which a burst wave having a sine curve with a cycle number of 4 at a frequency of $250 \mathrm{kHz}$ and $\pm 5 \mathrm{~V}$ is input to a power amplifier from a waveform generator. Next the electric signal amplifies to a $\pm 75 \mathrm{~V}$ burst wave with a power amplifier and then input to the EMAT for transmission. The output of the receiving EMAT is amplified by a preamplifier with $40 \mathrm{~dB}$ amplification, then is amplified with a maximum of $40 \mathrm{~dB}$ amplification and filtered with a frequency window of $100 \mathrm{kHz}$ to $500 \mathrm{kHz}$ by the discriminator.

The signal is observed and recorded by an oscilloscope. Analysis is then done using a PC. 


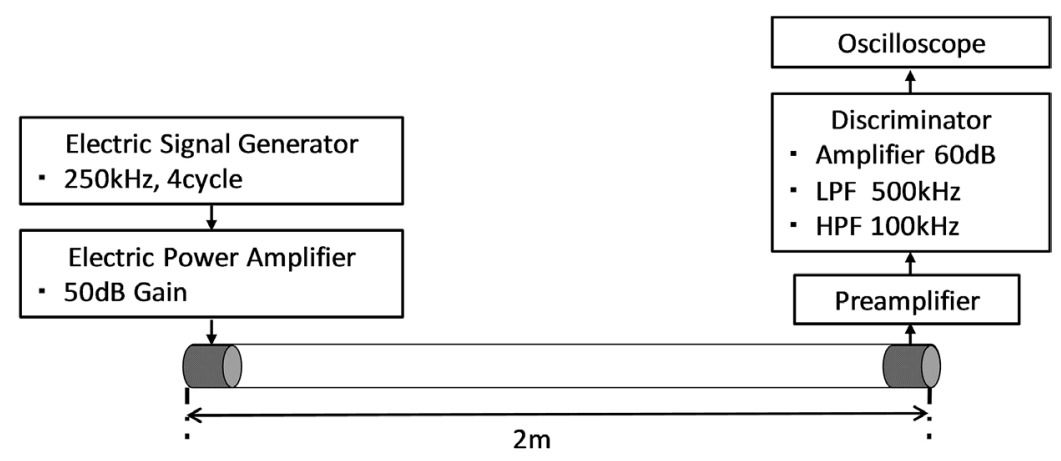

Figure 5. Overview of the experimental system.

When the EMAT for the longitudinal vibration mode guided wave and the EMAT for the transverse vibration guided wave were individually evaluated, the EMAT for the transmission and reception was installed on both ends of a 21.1 $\mathrm{mm}$ outer diameter $\times 2.8 \mathrm{~mm}$ thickness $\times 2 \mathrm{~m}$ length pipe. In the final flaw evaluation test, a transmission and reception EMAT were installed at one end of a $21.1 \mathrm{~mm}$ outer diameter $\times 2.8 \mathrm{~mm}$ thickness $\times 1 \mathrm{~m}$ long pipe, and evaluated the reflected signal from an artificial machined defect at the middle of the pipe.

\section{EMAT for Longitudinal Vibration Mode Guided Wave}

\subsection{Basic Structure}

Figure 6 shows the basic structure of the longitudinal vibration mode guided wave transmit ting/receiving EMAT. The bias magnetic field by the permanent magnet and the dynamic magnetic field by the electromagnetic induction coil (EM-coil) in the axial direction of the pipe induces a magnetostrictive vibration in the axial direction of the pipe. It becomes a source of the longitudinal vibration mode guided wave. In practice, a steel bar is installed at the middle of two permanent magnets which are placed at opposite polarity. The EM-coil is wound around the steel bar. As a result of preliminary experiments, the permanent magnet used had a cylindrical shape with $14 \mathrm{~mm}$ diameter and $15 \mathrm{~mm}$ length. The magnetic flux density on the surface in the axial direction was about 520 $\mathrm{mT}$. The number of turns of the EM-coil was 30, and its diameter was $0.2 \mathrm{~mm}$. The two magnets were connected to the iron bar, and the distance was experimentally determined because the magnitude of the magnetostrictive vibration greatly differs depending on the magnitude of the magnetic flux added to the surface of the test material, and there is a maximum value as shown in Figure 6(b). The magnitude of the magnetic field that reaches the maximum value varies depending on the material, but in the case of steel, it is often from 50 to 100 $\mathrm{mT}$. Therefore, we aimed to obtain the maximum magnetostrictive vibration by adjusting the distance between both magnets.

\subsection{Determination of Distance between Both Magnets}

Figure 7(a) shows the basic structure of the evaluated EMAT, and Figure 7(b) is 
an external view. Figure 7(c) shows the relationship of the distance between the magnets and the axial magnetic flux density on the EM-coil position. As a result, as shown in Figure 7(d), the transmitted received signal has the maximum signal amplitude at the distance of $5 \mathrm{~mm}$. This result is consistent with the fact that the magnetostriction variation with respect to the bias magnetic field of steel becomes a maximum at from 50 to $100 \mathrm{mT}$ as shown in Figure 6. Figure 7(e) shows the received signal waveform when the distance between the two magnets is $1 \mathrm{~mm}$, and Figure 7(f) shows the received signal waveform when the distance is $5 \mathrm{~mm}$. Both the signal amplitude and $\mathrm{S} / \mathrm{N}$ of the signal have been improved about 4 times.

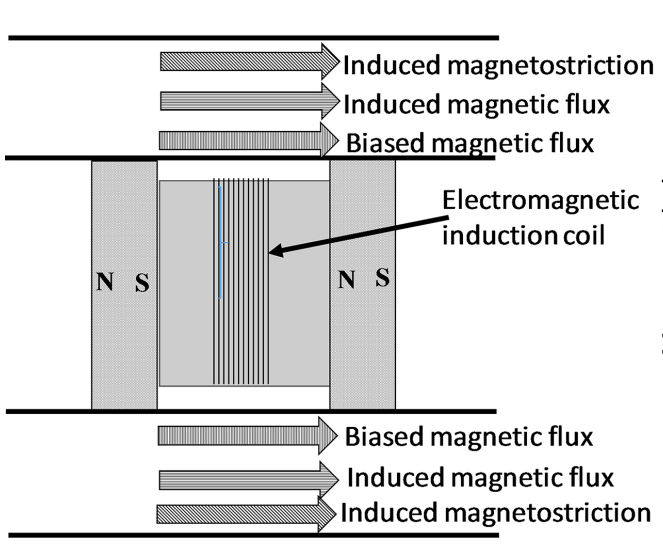

(a) Basic structure for $\mathrm{L}(0,2)$-mode EMAT

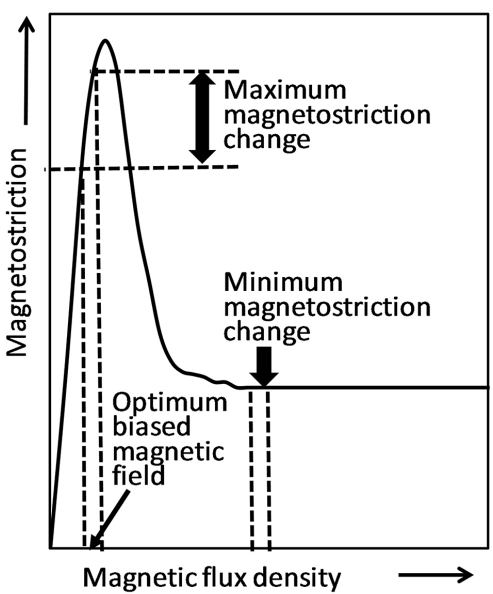

(b) Magnetic flux density and Magnetostriction

Figure 6. Basic structure for longitudinal vibration mode guided wave EMAT and generation mechanism to obtain the best magnetostrictive vibration.

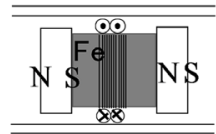

(a) Basic structure

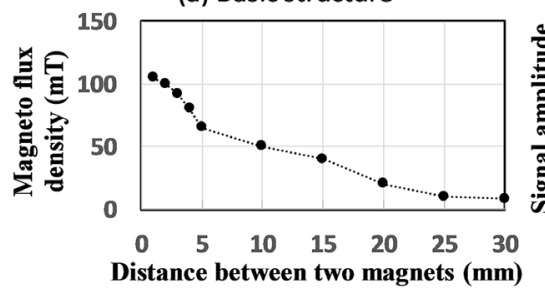

(c) Distance and Magnetic flux density

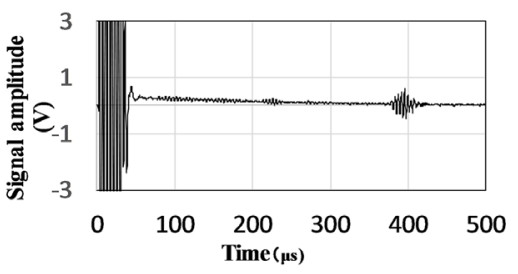

(e) Received signal waveform (1mm)

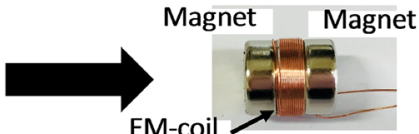

(b) External view

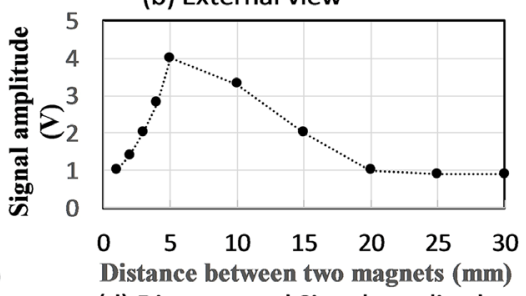

(d) Distance and Signal amplitude

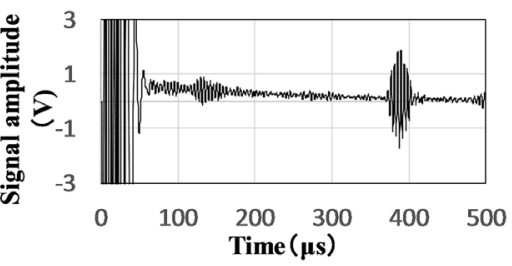

(f) Received signal waveform $(5 \mathrm{~m})$

Figure 7. Process to get the optimal EMAT for longitudinal vibration mode guided waves. 


\subsection{Acoustic Isolation Effect the Transmission/Reception EMAT}

First, when the integrated transmission/reception EMAT is directly connected as shown in Figure 8(a), that is, when the distance is $0 \mathrm{~mm}$ as shown in Figure 8 (d), a fixed noise signal is mixed in the received signal waveform as shown in Figure $7(\mathrm{e})$. No signal reflected from the other end of the pipe could then be confirmed. Therefore, as shown in Figure 8(b), the transmission and reception EMATs were separated, and the thickness of the insulation sheet was changed between the transmitter and the receiver EMAT. Figure 8(c) is an external view. Figure 8(e) shows the received signal waveform without the insulation sheet and Figure 8(f) shows the received signal waveform when the $2 \mathrm{~mm}$ thick insulating sheet was inserted, which was able to remove most of the mixed noise signal. The transmitted signal around $360 \mu$ s can be detected with a sufficient $S / N$. This result means that the transverse waves generated in the transmitting EMAT reach the receiving EMAT directly through the permanent magnet, unless the transmitting EMAT and the receiving EMAT are sufficiently acoustically isolated. This is a necessary consideration when it is necessary to transmit and receive only guided waves propagating on the pipe surface.

\subsection{Effect of the Multi-Channel EMAT}

With respect to the basic structure of Figure 9(a), it was confirmed whether the sensitivity can be improved by forming the transmission side into two channels or the reception side into two channels as shown in Figure 9(b) which is a diagram for the case of two channels on the receiving side. Figure 9 (c) is an external view of the multichannel EMAT. As a result of the evaluation, as shown in Figure $9(\mathrm{~d})$, it was confirmed that the signal amplitude was increased by about $50 \%$ when the receiving side had two channels. Figure $9(\mathrm{e})$ shows the signal

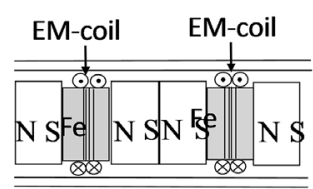

(a) Basic structure

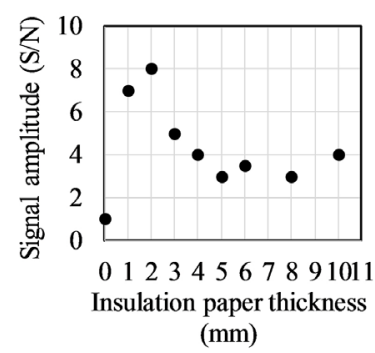

(d) The thickness of the insulation sheet and the received signal amplitude

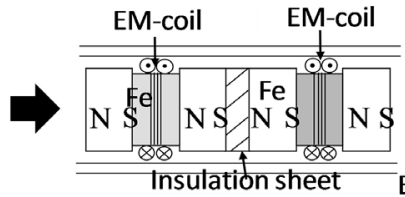

(b) Insulation sheet was inserted between the both EMATs

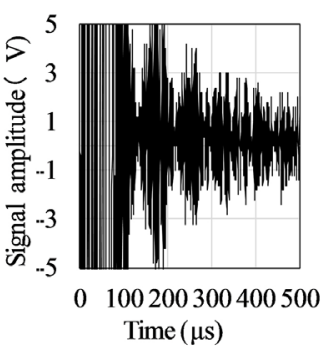

(e) The signal waveform by the basic structure

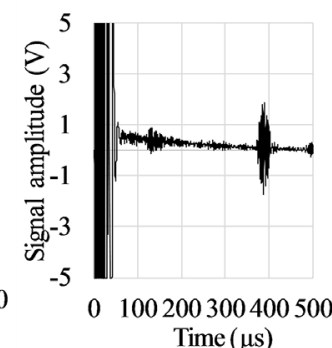

(f) The signal waveform using $2 \mathrm{~mm}$ insulation sheet

Figure 8. Improvement of the EMAT for longitudinal vibration mode guided waves which are connected to the transmitter EMAT and the receiver EMAT. 


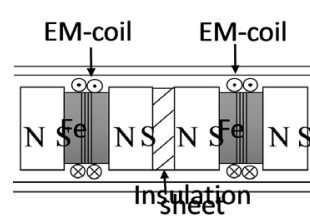

(a) Basic structure

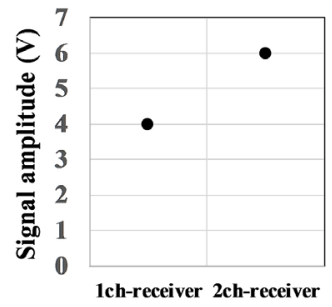

(d) The number of the receiverEMAT and signal amplitude

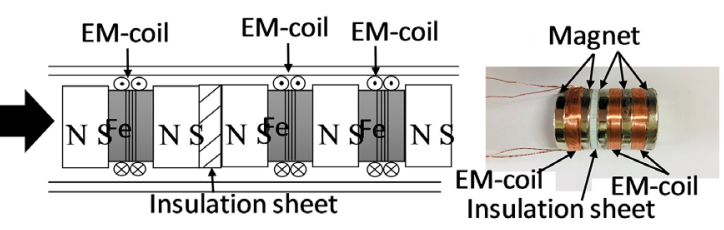

(b) 1ch-transmitter, 2ch-receiver

(c) External view

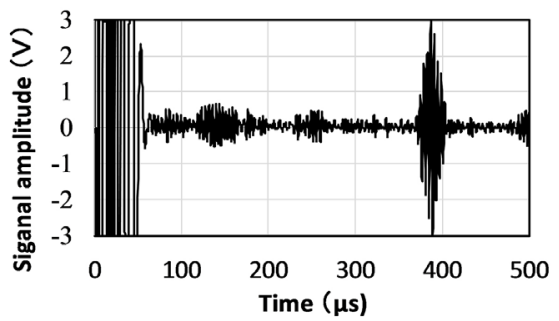

(e) The signal waveform using 2ch-receiver

Figure 9. Improvement of the EMAT for longitudinal vibration mode guided waves using multichannel EMAT.

waveform using the two-channel receiver-EMAT. This result shows that even if the transmission EMAT has multiple channels, it has no effect unless phase matching is obtained, and it is difficult to achieve phase matching. In addition, it is considered that it is in principle necessary to match the phases on the receiving EMAT as well, but it became clear that the conditions are not as severe as on the transmitting EMAT.

The distance attenuation coefficient was evaluated by changing the installation position in the pipe using the L-mode EMAT with the structure shown in Figure $7(\mathrm{c})$. The distance attenuation coefficient was $0.0023 \mathrm{~dB} / \mathrm{mm}$. This means that the signal amplitude was reduced by half at the traveling distance of about $2.4 \mathrm{~m}$. Since the $\mathrm{S} / \mathrm{N}$ of the signal at a distance of $2 \mathrm{~m}$ can be evaluated as 15 or more, it can be determined that the $\mathrm{S} / \mathrm{N}=2$ can be maintained even if the propagation distance becomes greater than about $10 \mathrm{~m}$.

\section{EMAT for Transverse Vibration Mode Guided Wave}

\subsection{Basic Structure}

As the EMAT for the transverse vibration mode guided wave, the SH plate wave EMAT for the flat thin plate shown in Figure 10 was referenced. The SH plate wave has a component that vibrates in the plane perpendicular to the traveling direction along the plate surface [14]. Therefore, using an array of permanent magnets whose periodic magnetization direction changes, the Lorentz force is generated by interaction with the alternating current flowing through the EM-coil placed under the magnet. The periodically changeable Lorenz force becomes the source of the SH-plate wave which vibrates in the plane and at the vertical direction to the traveling direction. Thus, an EMAT having the structure shown in Figure 11(a) was developed so that this structure could be directly realized inside the pipe. The magnet used was a neodymium type and arc-shaped magnet 

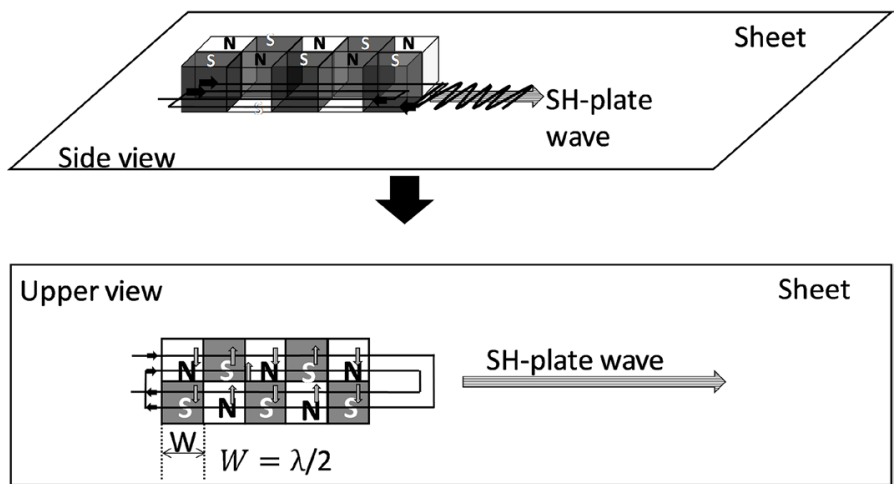

Sheet

Figure 10. Basic structure of an SH wave-EMAT for a thin plate.

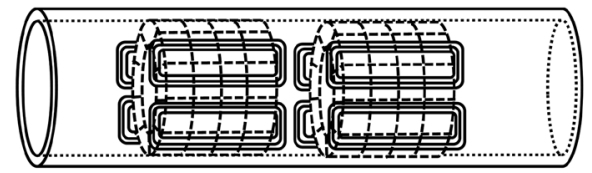

(a) Basic structure

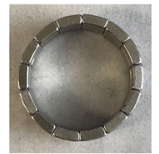

(b) Front view of the magnets

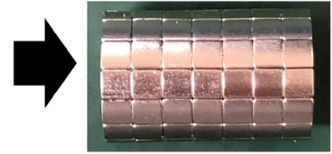

(c) Side view of the magnets

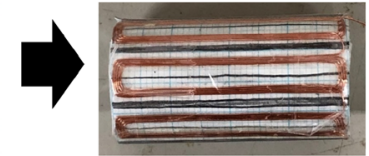

(d) Side view -EM-coil on the magnets-

Figure 11. Basic structure for transverse vibration mode guided wave traveling in axis direction of a pipe.

having an outer diameter of $15.0 \mathrm{~mm}$, an inner diameter of $10.0 \mathrm{~mm}$, a depth of $10 \mathrm{~mm}$, and a $25^{\circ}$ angle. Twelve pieces were connected in the circumferential direction to form a cylindrical shape and inserted into the pipe. Figure 11(b) is an external view of the magnet portion, and Figure 11(c) is a side view of the magnet portion. Figure 11(d) is a side view of the EM-coil arranged on the magnet. A cylinder array in the circumferential direction was defined as one row, and the influence of the number of rows was also examined. The EM-coils were arranged above the N-pole and S-pole in the circumferential direction, formed in an elliptical shape elongated in the axial direction, and arranged in six rows in the circumferential direction. As a result of a preliminary study, the specifications of each EM-coil was 4 turns and $100 \mathrm{~mm}$ in axial length.

Actually, it has been compared experimentally several structures such as a type that uses the magne tostriction effect to transmit and receive T-mode guided waves from the inside of the pipe, but the Lorentz force type EMAT using a periodic magnet has been proven that could detect a bigger received signal waveform by a T-mode guided wave.

\subsection{Effect of the Number of Magnet Rows}

Figure 12(a) shows the relationship between the number of columns and the trans mitted received signal amplitude. The best result was obtained using five rows. 


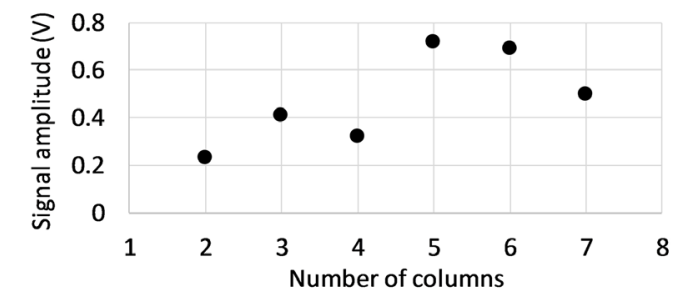

(a) Number of columns and signal amplitude

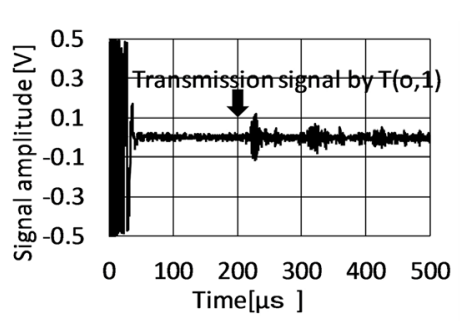

(b) Received signal waveform -Number of columns is 2-

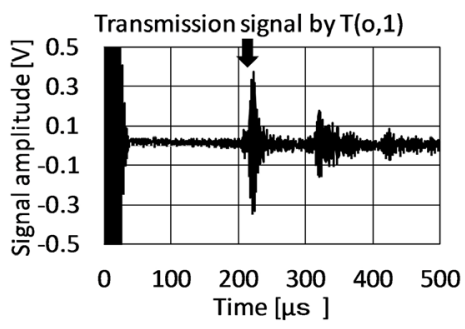

(c) Received signal waveform -Number of columns is 5 -

Figure 12. Determination of the number of magnet rows to obtain the best signal.

Figure 12(b) shows the received signal waveform in the case of two columns, and Figure 12(c) shows the received signal waveform in the case of five columns. Regarding the number of rows of permanent magnets in the circumferential direction, it was initially thought that an even number of rows with positive and negative vibration directions would be effective. However, the experimental results show that the odd columns are valid instead. Perhaps due to manufacturing accuracy, the spacing of the Lorentz force generated between adjacent magnets could not be perfectly matched to the half-wavelength of the T-mode guided wave, so the effect of increasing the in-phase vibration direction is more remarkable. The optimum value for the number of rows exists because the area of the electromagnetic induction coil that covers it increases as the number of rows increases. That is, it is considered that the impedance of the electromagnetic induction coil increases.

The distance attenuation coefficient was evaluated by changing the installation position in the pipe using the T-mode EMAT with the structure shown in Figure $7(\mathrm{c})$. The distance attenuation coefficient was is $0.0030 \mathrm{~dB} / \mathrm{mm}$. This indicated that the signal amplitude becomes half at the traveling distance of about $2 \mathrm{~m}$. The $\mathrm{S} / \mathrm{N}$ of the received signal at a distance of $1 \mathrm{~m}$ is about 10 , and the distance where $\mathrm{S} / \mathrm{N}=2$ then is considered to be less than $5 \mathrm{~m}$. A further improvement in the sensor performance is required in the future.

\section{Evaluation of the Flaw Detection Ability}

As shown in Figure 13, an EMAT for a longitudinal mode guided wave and an EMAT for a transverse mode guided wave were connected to perform an experiment for evaluating the detectability of artificial flaws. However, since there is only one electric drive device, the evaluation was performed by alternately driv- 
ing the EMAT for the longitudinal mode guided wave and the EMAT for the transverse mode guided wave.

Figure 14 shows the relationship between the depth of the notch flaw and the reflected signal amplitude when the notch flaws are formed in the circumferential direction around of the pipe. In both modes, a notch flaw having a depth of about $0.2 \mathrm{~mm}$ with respect to a thickness of $2.8 \mathrm{~mm}$, that is, a depth of about $10 \%$ or less with respect to the wall thickness was detected. In the case of the longitudinal mode guided wave mode, the reflectance is higher at any depth with respect to the ratio of the notch depth to the wall thickness. In the case of the transverse mode guided wave mode, the correspondence is relatively good. However, since the signal amplitude was originally low, the reproducibility was also poor. Figure 15 shows the relationship between the circumferential width of the notch flaw and the reflected received signal when the notch flaw is machined in the axial direction. In the longitudinal vibration mode guided wave, an axial notch with a circumferential width of $5 \mathrm{~mm}$ was detected. However, the transverse vibration mode guided wave could only be detected when the circumferential width was $10 \mathrm{~mm}$ or more. In the case of the longitudinal vibration mode guided wave mode, the correspondence is relatively good. In the case of the transverse vibration mode guided wave mode, the correspondence is not clear. In addition, since the signal amplitude was originally low, the reproducibility was also poor.

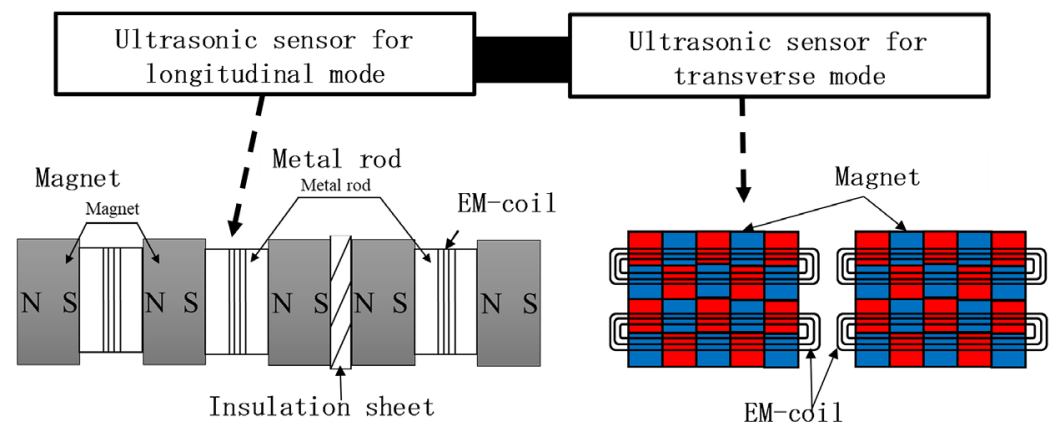

Figure 13. Overview of the prototype ultrasonic probe connected to the longitudinal vibration mode guided wave and the transverse vibration mode guided wave.

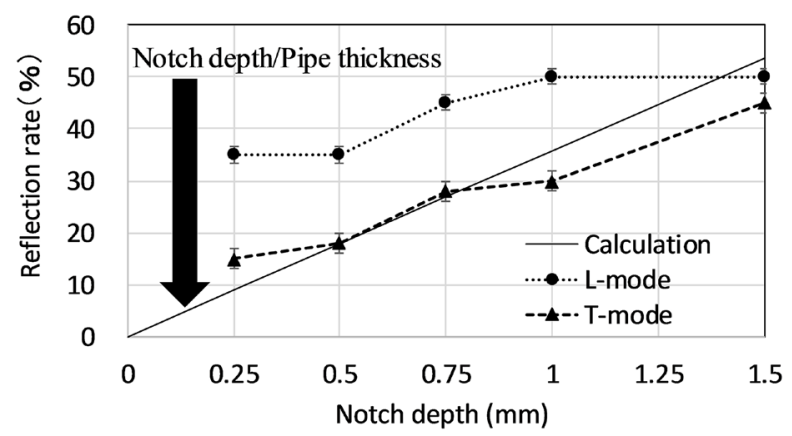

Figure 14. Relationship between the notch depth and reflection rate using reflected received signal from the notch all around a pipe. 


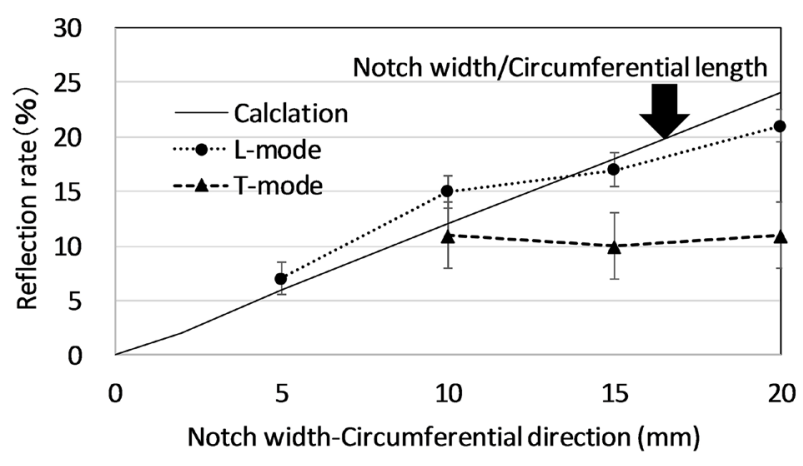

Figure 15. Basic structure for transverse vibration mode guided wave traveling in axis direction of a pipe.

\section{Conclusions}

For the purpose of the non-destructive inspection of any heat exchangers used in power plants, etc., an internal ultrasonic probe for steel pipes with diameters of $21.7 \mathrm{~mm}$ and $2.8 \mathrm{~mm}$ has been developed. The developed ultrasonic probe is based on the electromagnetic acoustic transducer (EMAT). It is assumed that it connects the EMAT for the longitudinal vibration mode guided wave $(\mathrm{L}(0,2))$ and the EMAT for the transverse vibration mode guided wave $(\mathrm{T}(0,1))$.

1) The longitudinal vibration mode guided wave EMAT is a type that uses the magnetostriction effect to generate the guided wave. In that case, it became clear that it was essential to optimize the distance between the two magnets to obtain the optimum biased magnetic field and to acoustically insulate between the EMAT for transmission and the EMAT for reception. It was also revealed that the propagation distance was about $10 \mathrm{~m}$.

2) For the transverse vibration mode guided wave, referring to the $\mathrm{SH}$ plate wave EMAT for thin plates, the number of rows of magnets in the axial direction was experimentally evaluated, and 5 rows were selected. It was also found that the propagation distance was about $5 \mathrm{~m}$.

3) Combining the longitudinal vibration mode EMAT and the transverse vibration mode EMAT, the relationship between the notch depth and the reflected signal amplitude was constant in both modes, but the correlation was higher in the transverse vibration mode. The correlation between the circumferential width of the axial notch and the reflected signal amplitude was found in the longitudinal vibration mode, but not in the transverse vibration mode.

In the future, we will develop an ultrasonic probe that improves the detection sensitivity of the transverse vibration mode guided waves and utilizes the frequency dependence of the vibration distribution with longitudinal mode guided waves.

\section{Conflicts of Interest}

The authors declare no conflicts of interest regarding the publication of this paper. 


\section{References}

[1] Tang, X.J. and Yan, D.C. (1997) Pipeline Leakage Detection Method and Instrument Based on Neural Network. Journal of Peking University, 33, 319-327.

[2] Fuchs, H.V. and Riehle, R. (1999) Ten Years of Experience with Leak Detection by Acoustic Signal Analysis. Applied Acoustics, 33, 1-19.

https://doi.org/10.1016/0003-682X(91)90062-J

[3] Gao, F.Q. (1998) The Necessity of Pipeline Inspection. Pipeline Technology and Equipment, 14, 40-42.

[4] Fang, H. (2000) A New Method for Magnetic Leakage Detection of Steel Tubes. Journal of Iron and Steel Research, 12, 50-54.

[5] Chen, H.B. and Tu, Y.Q. (2000) A Review of Leakage Detection Methods for Oil Pipeline. Pipeline Technology and Equipment, 22, 38-41.

[6] Rang, Q.F. (2010) Classification of Non-Destructive Testing Methods and Brief Introduction of Their Characteristics. Non-Destructive Testing, 21, 75-80.

[7] Li, J. and Rose, J.J. (2006) Natural Beam Focusing of Non-Axisymmetric Guided Waves in Large-Diameter Pipes. Ultrasonics, 44, 35-45. https://doi.org/10.1016/j.ultras.2005.07.002

[8] Thompson, R.B. (1997) Experiments in the Use of Guided Ultrasonic Waves to Scan Structures. Review of Progress in Quantitative NDE, 16, 121-128.

https://doi.org/10.1007/978-1-4615-5947-4_16

[9] Lowe, M.J.S., Alleyne, D.N. and Cawley, P. (1998) Defect Detection in Pipes Using Guided Waves. Ultrasonics, 36, 147-154. https://doi.org/10.1016/S0041-624X(97)00038-3

[10] Thompson, R.B. (1973) A Model for the Electromagnetic Generation and Detection of Rayleigh and Lamb Wave. IEEE Transaction on Sonics and Ultrasonics, 20, 340-346. https://doi.org/10.1109/T-SU.1973.29770

[11] Murayama, R. (2002) Latest Trend and Basics of an Electromagnetic Acoustic Transducer Method. Journal of the Japanese Society for Non-destructive Inspection, 51, 62-67. (in Japanese)

[12] Hirao, M. and Ogi, H. (2003) Coupling Mechanism. In: EMATs for Science and Industry. Springer, Boston, MA, 13-38.

https://doi.org/10.1007/978-1-4757-3743-1 2

[13] Hayashi, T. (2007) Guided Wave Dispersion Curves Derived with a Semi-Analytical Finite Element Method and Its Applications to Nondestructive Inspection. Proceedings of Symposium on Ultrasonic Electronics, 28, 351-352. (in Japanese)

[14] Ribichini, R., Cegla, F.B., Nagy, P.B. and Cawley, P. (2011) Study and Comparison of Different EMAT Configurations for SH Wave Inspection, IEEE Transaction on Ultrasonics Ferroelectrics and Frequency Control, 58, 2571-2581.

https://doi.org/10.1109/TUFFC.2011.2120 